\title{
Structural Design and Motion Mechanism of the Rope-Drive Snake-Like Robot
}

\author{
ZHAO Zhengyang ${ }^{1,2,3}$, GAO Junyao ${ }^{1,2,3}$, HUANG Chengzu ${ }^{1,2,3}$, WU Qianying ${ }^{1,2,3}$, WANG Cheng ${ }^{1,2,3}$, SU \\ Xuandong ${ }^{1,2,3}$, LIU Huaxin ${ }^{1,2,3}$, LI Xin ${ }^{1,2,3}$, LIU Yi $^{1,2,3}$ XU Zhe ${ }^{1,2,3}$ \\ ${ }^{1}$ Intelligent Robotics Institute, ${ }^{2}$ Key Laboratory of Biomimetic Robots and Systems, Ministry of Education, \\ ${ }^{3}$ State Key Laboratory of "Intelligent control of complex systems and decision" \\ School of Mechatronical Engineering, Beijing Institute of Technology, \\ 5 Nandajie, Zhongguancun, Haidian, Beijing, 100081, China \\ corresponding phone: 86-10-68913111; fax: 86-10-68915812; e-mail: gaojunyao@bit.edu.cn
}

\begin{abstract}
The characteristics of the snake with a variety of motion patterns and multi-redundancy and multi-degree of freedom make it have powerful motion ability and good environmental adaptability, can pass through obstacles that human can't. These advantages prompt us to study a new-type rope-drive snake-like robot based on the epidermis-drive. The snake-like robot uses modular design, with a remarkable versatility, economy and robustness. In this paper, we mainly design the structure of the rope-drive snake-like robot, and carry out a detail description of the drive mechanism, the drive principle and the yaw and pitch mechanism of the snake-like robot. And then study the motion mechanism of the rope-drive snake-like robot; finally verify the motion ability of the snake-like robot by experiments.
\end{abstract}

Keywords-rope-drive snake-like robot; structural design; motion mechanism

\section{INTRODUCTION}

In nature, snakes spread all over the earth, after millions of years of evolution, a variety of motion patterns and physiological characteristics enable them to adapt to a wide range of geographic and natural environment and make them be one kind of animals with the strongest obstacle performance in the world, and thus a variety of snake-like robots mimic the movement of the snake become research focus of the scientists, and there are fruitful results. Snake-like robot's motion mechanism and behavior are similar to biological snake, it can crawl in all kinds of rough, steep, rugged and complex terrain and can climb obstacles, therefore it has a broad application prospects in many fields, such as in radiant, dust, toxic environment and reconnaissance on the battlefield; It can also find the wounded in the ruins after the earthquake, landslide and fire and detect and clear the pipeline in narrow and dangerous environment.

Professor Hirose [1] studied snake from the perspective of biology for the first time. His most well-known result is summarized the serpenoid curve whose curvature changes in sinusoidal, he also explained why exert torque along the snake can generate forward force in principle. For structural reason, the snake-like robot can only achieve plane motion and cannot be applied to practical.

This research was supported in part by Beijing Physical Science Fund (Grant NO.4102054) and 111 Project (Grant NO.B08043)
Ryo Ariizumi, Hiroaki Fukushima and Fumitoshi Matsuno [2]- [4] developed a snake-like robot using screw drive mechanism. The robot is comprised of two types of screw drive units, i.e. "left" and "right" screw units, right and left units are connected alternatively from the head. The screw drive units are connected by 2-DOF active joints. Each actuator for pitch and yaw angles has a 90-degree range of movement. For a particular trajectory, the control system just need to give command to the head, the rest of the joints will be able to automatically calculate the trajectory of the previous joint and track. However, due to structural reason, its movement will result in stability issue and the movement efficiency is low.

Grzegorz Granosik and Johann Borenstein[5][6] developed a Omnitread snake-like robot with pneumatic joints and the whole body is covered with moving tracks, which make the robot has a strong power and enhance its ability of climbing obstacle, and various forms of movements can be achieved by adjusting the pneumatic joints instead of the serpenoid curve movement previous snake-like robots are used to adopt. However, the size and weight of the robot are relatively larger, and sundries can easily enter the robot's body.

In this paper, we design a new-type rope-drive snake-like robot [7]; the snake-like robot epidermis has motive power through specific mechanical design, and can simplify winding, twisting, tumbling and other movements. The robot with a small size, moves fast, and has strong practicability; it can solve the existing bottlenecks that are short of power, control difficulties and low obstacle performance in complex environment.

\section{STRUCTURAL DESIGN OF THE ROPE-DRIVE SNAKE-LIKE ROBOT}

The rope-drive snake-like robot adopts modular design concept, both the mechanical parts and the hardware and software components of the control system adopt modular design. Each module is the same of the structure and has almost the same circuit and control program. Modular design can simplify design, reduce cost, and ease maintenance. 


\section{A. The Structure of a Single Module}

A single module of the rope-drive snake-like robot is shown in Fig. 1, Fig. 2 is the internal view after removal of a cylindrical skeleton, and Fig. 3 shows the front view of the multi-groove pulley. Table 1 lists some key specifications of the rope-drive snake-like robot.

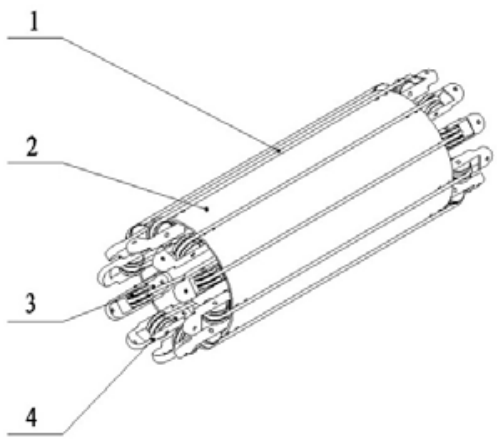

Fig.1. A single module

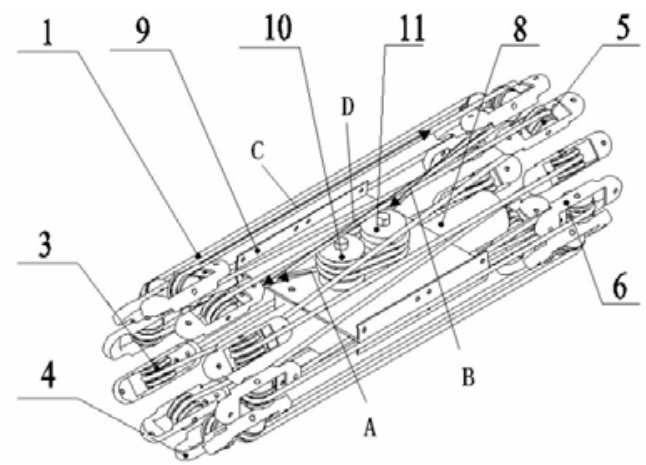

Fig.2. Internal view of the module

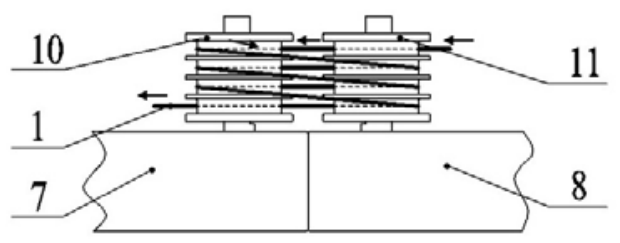

Fig.3.Front view of the multi-groove pulley

TABLE1.SPECIFICATIONS FOR ONE MODULE

\begin{tabular}{|c|c|}
\hline Weight(kg) & 2 \\
\hline Length(mm) & 300 \\
\hline Diameter(mm) & 114 \\
\hline Speed(m/s) & 0.1 \\
\hline
\end{tabular}

In Fig. 1, 2 and 3 ,1 represents rope, 2 represents skeleton, 3 represents front guide wheel, 4 represents front guide wheel bracket, 5 represents rear guide wheel, 6 represents rear guide wheel bracket, 7 represents front motor, 8 represents rear motor, 9 represents motor mounting bracket, 10 represents front multi-groove pulley, 11 represents rear multi-groove pulley.

As shown in Fig. 1 and Fig. 2, the module consists of rope, skeleton, guide wheels, guide wheel brackets and drive. One end of the skeleton has ten rotating front guide wheels, the other end has ten rotating rear guide wheels, the front guide wheel is fixed to the front guide wheel bracket, the rear guide wheel is fixed to the rear guide wheel bracket, the front and rear guide wheel bracket are fixed in the front and rear of the skeleton by screws, the drive is installed in the central location of the skeleton through screws.

\section{B. Drive Mechanism and Drive Principle}

Drive mechanism of the rope-drive snake-like robot is shown in Fig. 3, including two motors, motor mounting bracket, front and rear multi-groove pulleys. The multi-groove pulleys couple with the motor output shafts, the motors are fixed to the motor mounting bracket, the motor mounting bracket is fixed to the central location of the skeleton by screws.

The drive principle of the rope-drive snake-like robot is double multi-groove pulleys drive. Refer to Fig. 3, one end of the rope bypasses the first groove of the front multi-groove pulley via the first groove of the rear multi-groove pulley, after tilting a certain angle bypasses the second groove of the rear multi-groove pulley via the first groove of the front multigroove pulley, and then bypasses the second groove of the front multi-groove pulley via the second groove of the rear multi-groove pulley, and so on, the rope winds around into the first groove of the rear multi-groove pulley and winds around out of the fourth groove of the front multi-groove pulley.

Refer to Fig. 2, after winding around out of the fourth groove of the rear multi-groove pulley, the rope moves to the outer wall of the skeleton via the front guide wheel (C represents the direction of movement of the rope outside the skeleton), and then moves into the skeleton via the rear guide wheel (D represents the direction of movement of the rope within skeleton), and then forms a certain inclination with the axis of the skeleton, moves to the next front guide wheel, and so on, after bypassing every guide wheel, one end of the rope connects to the other end of the rope on the rear multi- groove pulley, finally using high-temperature fusion or glue mount together to form the two ends of the rope into a closed loop.

The motors drive the multi-groove pulley, so as to drive the rope, the rope drives the skeleton, after changing direction of movement by the guide wheels, the directions of movement of the rope winding on the outer wall of the skeleton are the same, the friction between the rope and the guide wheels and the multi-groove pulleys is big enough, and the relative sliding is small, then the rotation of the motors can transform to the movement of the skeleton.

As a key technology of the rope-drive snake-like robot, driven material that is rope is essential to choose. After a long period of exploration and research, we abandon the use of general rope, but choose a strong elasticity, toughness, wear 
resistance and easy welding PU round belt as driven material. The experiments show that the PU round belt can well meet the requirements.

\section{Yaw and Pitch Mechanism Design}

A single module cannot achieve a complex movement, to make the snake-like robot with 2D and 3D motion capabilities there must be a connecting device between modules. Joint of the Rope-drive snake-like robot consists of yaw and pitch mechanisms. See Fig. 4, connecting device includes connection bracket, fixed bearing, yaw motor and pitch motor. The motor is fixed with the drive motor referred to in the subsection B through the fixed bearing, the connection bracket is a cross - shaped, and one side connects with the yaw motor the other side connects with the pitch motor, the motors rotation can generate yaw and pitch movements. Modules add a connecting device is shown in Fig. 5.

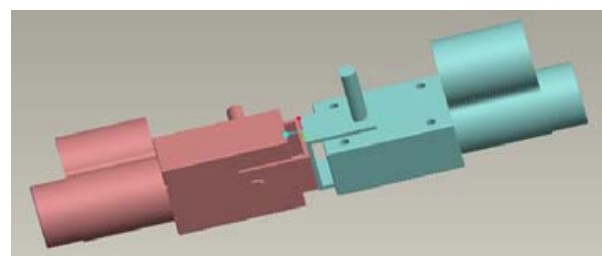

Fig.4. Yaw and pitch joints

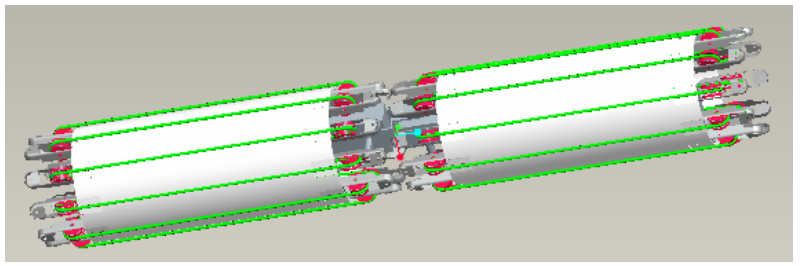

Fig.5. Modules with yaw and pitch joints

For purpose of calculation, the robot may be simplified as Fig. 6, Fig. 7.
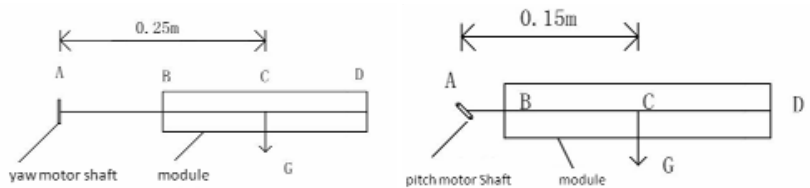

Fig.6. Torque calculation diagram of yaw module

Fig.7. Torque calculation diagram of pitch module

In above two figures, the segment $\mathrm{BD}$ represents the module of the snake-like robot, segment $A B$ represents yaw and pitch mechanisms, point $A$ represents the action point of output torque of the motor, point $C$ represents the center of gravity of the module. Ignoring the weight of segment $A B$, the mass of the module is $2 \mathrm{Kg}$, the length of segment $\mathrm{AC}$ is $0.25 \mathrm{~m}$, and the friction coefficient between the ground and the module is 0.4 .

Torque of yaw motor:

$T_{H} \geq F_{H} \times L_{A C}=G \times \mu \times L_{A C}=1.96 \mathrm{~N} \bullet \mathrm{m}$

and torque of pitch motor:

$T_{V} \geq G \times L_{A C}=2 \mathrm{Kg} \times 9.8 \mathrm{~N} / \mathrm{Kg} \times 0.15 \mathrm{~m}=2.94 \mathrm{Ngm}$

\section{The Oerall Sructure}

Fig. 8 shows the rope-drive snake-like robot, composed of six identical modules connected in series, the modules connect by the yaw and pitch joints, it can achieve 2D and 3D movements flexible, the total length is $2.05 \mathrm{~m}$ and the total weight is $12 \mathrm{Kg}$. Using active power supply, the speed of the robot can reach to $0.1 \mathrm{~m} / \mathrm{s}$.

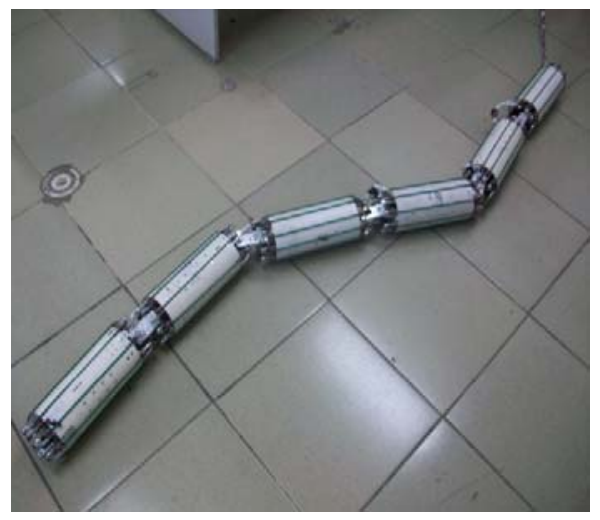

Fig.8.The rope-drive snake-like robot

\section{Motion MECHANISM OF THE ROPE-DRIVE SNAKE-LiKE ROBOT}

Each module of the rope-drive snake-like robot designed in this paper has a forward momentum, and all joints are active joints, the motion pattern is simple and flexible, the control method is relatively simple compared to the traditional sine wave motion of snake-like robot, and there is no need to establish a database for each motion pattern, avoiding a lot of complicated calculations. Each joint can be individually controlled to form different gestures, different gestures correspond to different paths; the snake-like robot can adjust gesture according to the relationship between the gestures and the paths, so that it can bypass obstacles smoothly and reach the target point.

Follow the head control algorithm [8] [9] is the most simple and effective control algorithm currently known. The basic principle is: given the position and gesture of the head, module behind follows the position and gesture of the head successively, thus the trajectory of each module can be fitted into a curve.

Based on follow the head control algorithm, in an environment with obstacles, as long as the head can get through, module behind can get through by following the head. Take the joint angle $\varphi_{n-1}$ of the head as a reference, delay for $\Delta t$, the movement of the head spreads backward, set the distance between two adjacent joints as $2 l$, then $\varphi_{i}(s=s+2 l)=\varphi_{i-1}(s)$, set the speed of spread as the movement speed of snake-like robot that is $V$, then $\Delta t=2 l / v$,thereby the expression of each joint angle can be expressed as $\varphi_{i}(t)=\varphi_{n-1}(t-(n-i-1) \Delta t)$, $i=1,2 \cdot \cdots \cdot n-2$. 
In this way, the head guidance control method only need to record the head rotation angle, and then hand on the angle to the next joint.

Without loss of generality, take the three links snake-like robot as an example to analyze the turning motion, as shown in Fig. 9.

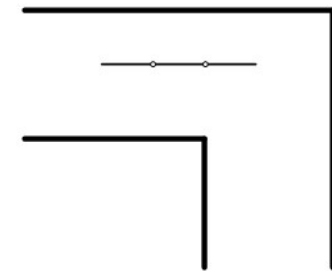

Fig.9(a) Initial state

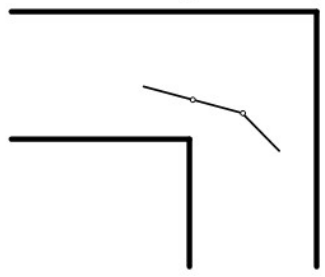

Fig.9(c) before the rotation of the second link.Fig.9 (d) after the rotation of the second link

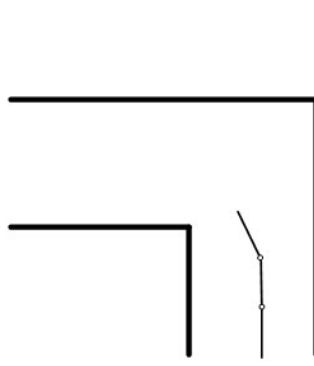

Fig.9(e) The head turns $-\varphi$

Fig.9(f) the second link turns $-\varphi$

Initial state as shown in Fig.9 (a), the head turns $\varphi$ to the right at the time $t_{1}$, the second link turns at the time $t_{1}+\Delta t$, Fig.9(c) is before the rotation and Fig.9 (d) is after the rotation. The angle between the head and the second link must be zero if the robot wants to move straightly after turning, so the head needs to turn $-\varphi$ at the time $t_{1}+\Delta t$, see Fig.9 (e). And the second link also needs to turn $-\varphi$ at the time $t_{1}+2 \Delta t$ after the robot bypassing the corner, as shown in Fig.9 (f).

The experiment shows that this control method is simple and effective; the snake-like robot can turn and avoid the obstacles, it is appropriate for spacious environment. In order to facilitate movement, manual intervention is allowable during the movement to adjust the shape of the robot at any time.

\section{EXPERIMENTAL ANALYSIS}

\section{A. Experiment of Rectilinear Motion}

The rectilinear motion is the simplest form of motion and is the best form to reflect the motion capacity of the snake-like robot. Each joint angle sets as zero when the snake-like robot is moving along a straight line, each module participates in the motion at the same speed, and the experimental data are shown in Table 2. The table shows the straight-line velocity of the robot is about $0.1 \mathrm{~m} / \mathrm{s}$, which is able to meet the practical requirement.

TABLE 2.DATA OF RECTILINEAR MOTION

\begin{tabular}{|c|c|c|c|}
\hline No. & Distance(m) & Time(s) & Velocity(m/s) \\
\hline 1 & 0.816 & 7.40 & 0.1103 \\
\hline 2 & 0.816 & 8.03 & 0.1016 \\
\hline 3 & 0.816 & 8.24 & 0.990 \\
\hline Mean & & & 0.1034 \\
\hline
\end{tabular}

\section{B. Experiment of Climbing Motion}

The climbing ability is also an important aspect of the motion capacity of the snake-like robot, the experimental condition of the climbing motion of the rope-drive snake-like robot is shown in Fig. 10, and Table 3 shows the experimental results. It is shown that the snake-like robot can climb a 30 degrees slope easily and smoothly.

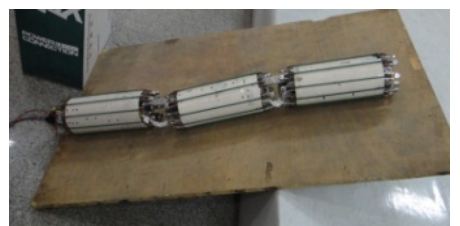

Fig.10 experimental condition of the climbing motion

TABLE 3 EXPERIMENTAL RESULTS OF THE CLIMBING MOTION

\begin{tabular}{|c|c|}
\hline Gradient & Climbing status \\
\hline $20^{\circ}$ & passed \\
\hline $25^{\circ}$ & passed \\
\hline $30^{\circ}$ & passed \\
\hline
\end{tabular}

\section{Experiment of Turning Motion}

To form different gestures and achieve turn, obstaclecrossing and other movements, modules of the snake-like robot need to cooperate during the motion. In order to simplify the control, head guidance control method is proposed, that is only control the movement of the head, the other links imitate the movement successively, and the method has been described in detail in section III. We design a turning experiment in the L-type channel of the snake-like robot. The results are shown in Fig.11. Fig.11 (a) shows the snake robot enter into the channel, Fig.11 (b) is the state before the robot makes a turn, Fig.11 (c) shows the head completes the turn, Fig.11 (d) shows the second link turns a same angle after a delay time, Fig.11 (e) shows the head turns a reversed angle so that the robot can move straightly, Fig.11 (f) is the state that the second link turns a reversed angle. Thus the robot completes the turning movement and moves on. The experiment shows that this control method is simple and effective. 


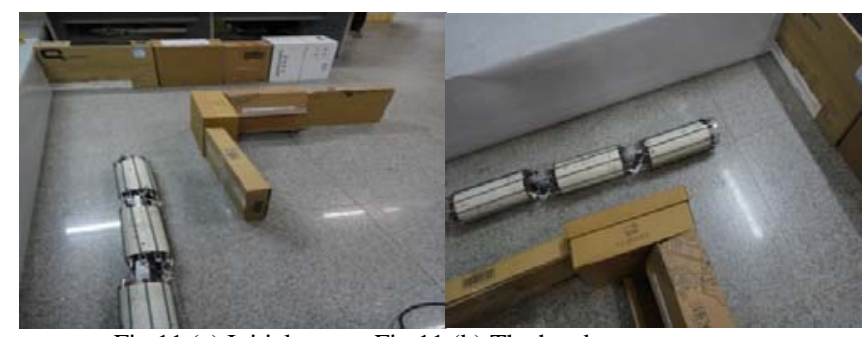

Fig.11 (a) Initial state Fig.11 (b) The head prepares to turn

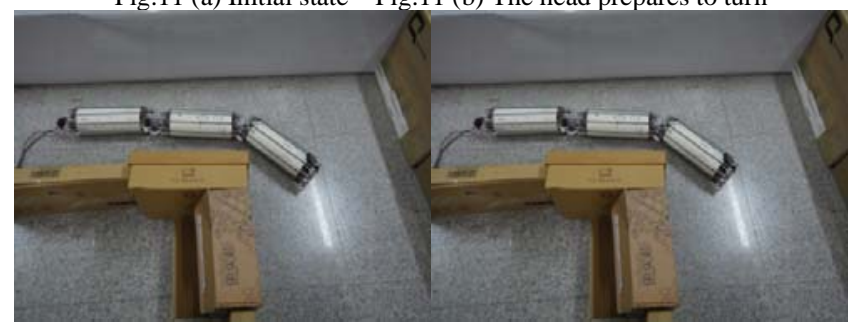

Fig.11(c) The head turns $\varphi$

Fig.11 (d) The second link turns $\varphi$

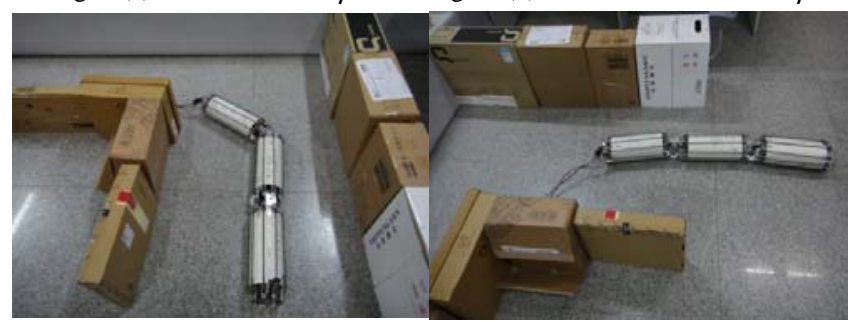

Fig.11 (e) The head turns - $\varphi \quad$ Fig.11 (f) The second link turn - $\varphi$

\section{Experiment of Ditch Crossing}

With the slender body, the snake-like robot can span a wide ditch. Fig.12 shows the process of crossing the ditch of the snake-like robot. The strategy is: the head must reach to the far-end of the ditch before the center of gravity of the robot reach the near-end of the ditch. The experimental condition of the ditch crossing is as shown in Fig. 12. In theory, the snakelike robot can get through a ditch whose width equals to a half length of the robot as long as the joints have sufficient stiffness. The experiment verifies this theory.

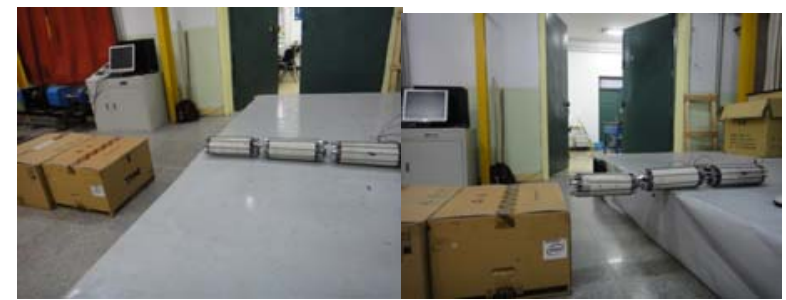

Fig.12 (a) Prepare to cross the ditch Fig.12 (b)The head is arriving at the far-

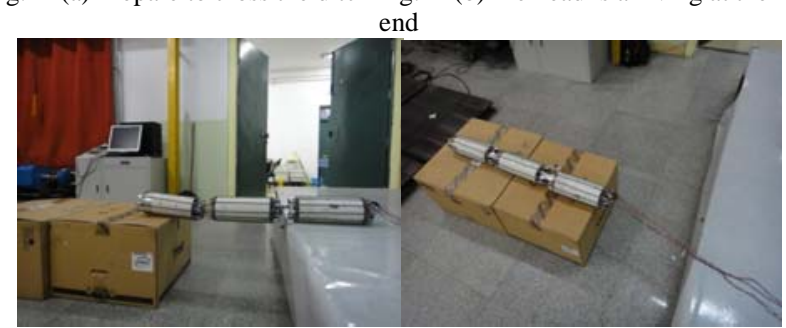

Fig.12 (c)The head arrives at the far-end Fig.12 (d)Get through the ditch

\section{CONCLUSIONS AND FUTURE RESEARCH DIRECTION}

Based on the idea of epidermal-drive we design a ropedrive snake-like robot, which has better terrain adaptability and better development prospects compared to traditional snake-like robot relied on twisting to forward.

The main achievement of this paper is to develop a new type of rope-drive snake-like robot, based on the principle of epidermal-drive, the whole body of the robot has power, and the obstacles will provide driving force for the rope-drive snake-like robot rather than resistance. Rope-drive snake-like robot has a strong athletic ability is verified by experiments.

At present, the velocity of the rope-drive snake-like robot is not fast, reduce weight and increase flexibility are the directions of future efforts. And the snake-like robot uses a wired mode power supply, limiting its range of applications. In the future, we plan to provide power by fixing one battery in the last link. Snake-like robot needs for sophisticated sensors and the ability of the independent movement to adapt to the complex and changing environment, which requires quality control, this paper just makes a basic research in this regard. To achieve full autonomy movement is an important direction for future snake-like robot research.

\section{REFERENCES}

[1] S.Hirose, Biologically Inspired Robot-Snake-like locomotors and manipulators. Oxford University Press,1993.

[2] S. Satomura, M. Hara, H. Fukushima, T. Kamegawa, H. Igarashi, and F. Matsuno: "Control of a Snake-Like Robot Using the Screw Drive Mechanism” Proc. of IEEE International Conference on Robotics and Automation, 2007, pp. 3883-3888.

[3] Ryo Ariizumi, Hiroaki Fukushima, and Fumitoshi Matsuno:”Front-UnitFollowing Control of a Snake-like Robot Using Screw Drive Mechanism Based on Past Velocity Commands", Proc. IEEE/RSJ International Conference on Intelligent Robots and Systems, 2011, pp. 1907-1912.

[4] Hiroaki Fukushima, Shogo Satomura, Toru Kawai, Motoyasu Tanaka, Tetsushi Kamegawa,and Fumitoshi Matsuno:”Modeling and Control of a Snake-Like Robot Using the Screw-Drive Mechanism", Proc. IEEE TRANSACTIONS ON ROBOTICS, 2012, pp. 1-14.

[5] Grzegorz Granosik, Malik G. Hansen and Johann Borenstein, The OmniTread serpentine robot for industrial inspection and surveillance[J]. Industrial Robot: 32/2 (2005) 139-148.

[6] Johann Borenstein,Malik Hansen, and Adam Borrell, The OmniTread OT-4 Serpentine Robot-Design and Performance[J], Journal of Field Robotics:24(7)(2007)601-621.

[7] GAO junyao, GAO xueshan, ZHU wei, ZHU jianguo, WEI boyu:” Design and Research of a New Structure Rescue Snake Robot with All Body Drive System”, Proceedings of IEEE International Conference on Mechatronics and Automation, 2008, pp. 119-124.

[8] K.U.Scholl, V.Kepplin, K.Berns, R.Dillmann:”Controlling a multi-joint robot for autonomous sewer inspection", Proc. Of IEEE International Conference on Robotics and Automation, 2000, pp. 1701-1706.

[9] B.Murugendran, A.Andreas Transeth, S.A.Fjerdingen:”Modeling and Path-following for a Snake Robot with Active Wheels", IEEE/RSJ International Conference on Intelligent Robots and Systems, 2009, pp. 3643-3650. 